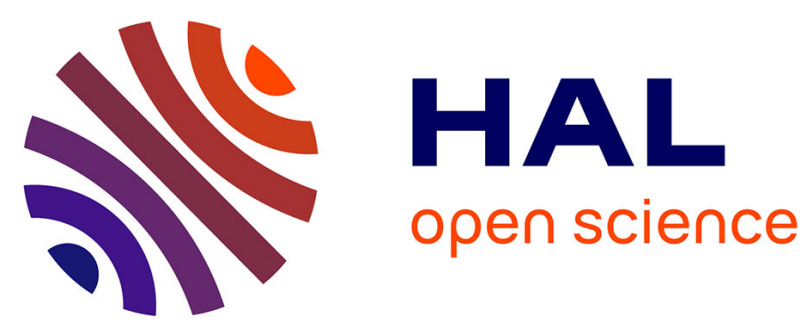

\title{
Coupled continuous-time random walks for fluid stretching in two-dimensional heterogeneous media
}

Marco Dentz, Daniel R. Lester, Tanguy Le Borgne, Felipe P.J. de Barros

\section{To cite this version:}

Marco Dentz, Daniel R. Lester, Tanguy Le Borgne, Felipe P.J. de Barros. Coupled continuous-time random walks for fluid stretching in two-dimensional heterogeneous media. Physical Review E , 2016, 94 (6), pp.061102(R). 10.1103/PhysRevE.94.061102 . insu-02442398

\section{HAL Id: insu-02442398 \\ https://hal-insu.archives-ouvertes.fr/insu-02442398}

Submitted on 10 Nov 2020

HAL is a multi-disciplinary open access archive for the deposit and dissemination of scientific research documents, whether they are published or not. The documents may come from teaching and research institutions in France or abroad, or from public or private research centers.
L'archive ouverte pluridisciplinaire HAL, est destinée au dépôt et à la diffusion de documents scientifiques de niveau recherche, publiés ou non, émanant des établissements d'enseignement et de recherche français ou étrangers, des laboratoires publics ou privés. 


\title{
Coupled continuous time random walks for fluid stretching in two-dimensional heterogeneous media
}

\author{
Marco Dentz \\ Spanish National Research Council (IDAEA-CSIC), 08034 Barcelona, Spain* \\ Daniel R. Lester \\ School of Engineering, RMIT University, 3000 Melbourne, Victoria, Australia \\ Tanguy Le Borgne \\ Geosciences Rennes, UMR 6118, Université de Rennes 1, CNRS, Rennes, France \\ Felipe P. J. de Barros \\ Sonny Astani Department of Civil and Environmental Engineering, University of Southern California, \\ 3620 S. Vermont Avenue, KAP 224B, Los Angeles, CA 90089, USA
}

(Dated: November 30, 2016)

\begin{abstract}
We study the relation between flow structure and fluid deformation in steady flows through two-dimensional heterogeneous media, which are characterized by a broad spectrum of stretching behaviors, ranging from sub- to superlinear. We analyze these behaviors from first principles, which uncovers intermittent shear events to be at the origin of subexponential stretching. We derive explicit expressions for Lagrangian deformation and demonstrate that stretching obeys a coupled continuous time random walk, which for broad distributions of flow velocities becomes a Lévy walk. The derived model provides a direct link between the flow and deformation statistics, and a natural way to quantify the impact of intermittent shear events on the stretching behavior.
\end{abstract}

\section{INTRODUCTION}

The deformation dynamics and stretching history of material fluid elements are fundamental for the understanding of hydrodynamic phenomena ranging from scalar dispersion, pair dispersion [1-3], mixing [4-9] and reaction [10-13] to the alignment of material elements [14] and the distribution of stress in complex fluids [15]. Fluid elements constitute the Lagrangian support of a transported scalar. Thus, their deformation histories determine the organization of the scalar distribution into lamellar structures [16-19]. Observed broad scalar concentration distributions are a manifestation of a broad distribution of stretching and compression rates and can explain intermittent patterns of scalar increment distributions $[16,17]$. The temporal scaling of the average elongation $\langle\ell(t)\rangle$ of material lines controls the decay of scalar variance, the effective kinetics of chemical reactions and the distribution of scalar gradients [20]. The mechanisms of linear stretching due to persistent shear deformation, and exponential stretching in chaotic flows have been well understood [20]. Observations of subexponential and non-linear fluid elongation [19, 21, 22], pair-dispersion $[1-3,23,24]$, and scalar variance decay $[25,26]$, however, challenge these paradigms and ask for new dynamic frameworks. As a consequence, different mechanisms of subexponential stretching have been proposed, including fractal or spiral mixing [e.g. 26], nonsequential stretching [e.g. 22], and modified Richardson

\footnotetext{
*marco.dentz@csic.es
}

laws [e.g. 25]. The dynamics of particle pair separation, for example, have been described using Levy processes and continuous time random walks [1, 3, 27]. Elongation time series for stretching in $d=2$ dimensional heterogeneous porous media flows have been modeled as geometric Brownian motions [8]. Most stochastic stretching models, however, do not provide relations between the deformation dynamics and the local Lagrangian and Eulerian deformations and flow structure. This means, the fluctuation mechanisms that cause observed algebraic stretching are often not known.

We focus here on fluid deformation in flows through heterogeneous porous media, which play a key role for the understanding of mixing and reaction processes in natural and engineered materials [28, 29]. For such flows, the mechanisms of (anomalous) particle dispersion have been the subject of intense theoretical and experimental studies [30-42]. The mechanisms of fluid stretching, however, are much less known. Here, we study the relation between velocity fluctuations and fluid deformation in non-helical steady flows through random media, specifically, we refer to steady $d=2$ pore-scale and $d=2$ and $d=3$ dimensional Darcy-scale flows in heterogeneous media [28]. The Darcy equation, which governs flow through porous media, implies that helicity is 0 in $d=3$ dimensions [43,44], and prohibits closed streamlines in $d=2$ [45]. Thus, flows through heterogeneous porous media are characterized by open streamlines, along which fluid particles may sample the full velocity spectrum [32, 46-48]. We derive here the mechanisms of subexponential and powerlaw stretching behaviors in such flows. To this end, we formulate Lagrangian deformation in streamline coordinates [49], which allows 
relating elongation to Lagrangian velocities and shear deformation from first principles. The consequences of this coupling are studied in the framework of a continuous time random walk (CTRW) [36, 50-52] that links transit times of material fluid elements to elongation through Lagrangian velocities. The CTRW framework has been used extensively for the quantification of particle motion in flows through heterogeneous media [36]. We develop here a CTRW framework to quantify the stretching of material fluid elements.

\section{FLUID DEFORMATION}

Our analysis starts with the equation of motion of a fluid particle in a steady spatially varying flow field. The particle position $\mathbf{x}(t \mid \mathbf{a})$ in the divergence-free flow field $\mathbf{u}(\mathbf{x})$ evolves according to the advection equation

$$
\frac{d \mathbf{x}(t \mid \mathbf{a})}{d t}=\mathbf{v}(t)
$$

where $\mathbf{v}(t)=\mathbf{u}[\mathbf{x}(t \mid \mathbf{a})]$ denotes the Lagrangian velocity. The initial condition is given by $\mathbf{x}(t=0 \mid \mathbf{a})=\mathbf{a}$. The particle movement along a streamline can be formulated as

$$
\frac{d s(t)}{d t}=v(t), \quad d t=\frac{d s}{v_{s}(s)},
$$

where $s(t)$ is the distance travelled along the streamline, $v(t)=|\mathbf{v}(t)|$ and the streamwise velocity is $v_{s}(s)=$ $|\mathbf{v}[t(s)]|$. With these preparations, we focus now on the evolution of the elongation of an infinitesimal material fluid element, whose length and orientation are described by the vector $\mathbf{z}(t)=\mathbf{x}(t \mid \mathbf{a}+\delta \mathbf{a})-\mathbf{x}(t \mid \mathbf{a})$. According to (1), its evolution is governed by

$$
\frac{d \mathbf{z}(t)}{d t}=\boldsymbol{\epsilon}(t) \mathbf{z}(t),
$$

where $\boldsymbol{\epsilon}(t)=\nabla \mathbf{u}[\mathbf{x}(t \mid \mathbf{a})]^{\top}=\nabla \mathbf{v}(t)^{\top}$ is the velocity gradient tensor. Note that $\mathbf{z}(t)=\mathbf{F}(t) \mathbf{z}(0)$ with $\mathbf{F}(t)$ the deformation tensor. Thus, $\mathbf{F}(t)$ satisfies Eq. (3) and the following analysis is equally valid for the deformation tensor. The elongation $\ell(t)$ is given by $\ell(t)=|\mathbf{z}(t)|$. We transform the deformation process into the streamline coordinate system [49], which is attached to and rotates along the streamline described by $\mathbf{x}(t \mid \mathbf{a})$,

$$
\mathbf{x}^{\prime}(t)=\mathbf{A}^{\top}(t)[\mathbf{x}(t)-\mathbf{x}(t \mid \mathbf{a})],
$$

where the orthogonal matrix $\mathbf{A}(t)$ describes the rotation operator which orients the $x_{1}$-coordinate with the orientation of velocity $\mathbf{v}(t)$ along the streamline such that $\mathbf{A}(t)=[\mathbf{v}(t), \mathbf{w}(t)] / v(t)$ with $\mathbf{w}(t) \cdot \mathbf{v}(t)=0$ and $|\mathbf{w}(t)|=v(t)$. From this, we obtain for $\mathbf{z}^{\prime}(t)=\mathbf{A}^{\top} \mathbf{z}(t)$ in the streamline coordinate system

$$
\frac{d \mathbf{z}^{\prime}(t)}{d t}=[\mathbf{Q}(t)+\tilde{\boldsymbol{\epsilon}}(t)] \mathbf{z}^{\prime}(t),
$$

where we defined $\tilde{\boldsymbol{\epsilon}}(t)=\mathbf{A}^{\top}(t) \boldsymbol{\epsilon}(t) \mathbf{A}(t)$ and the antisymmetric tensor $\mathbf{Q}(t)=\frac{d \mathbf{A}^{\top}(t)}{d t} \mathbf{A}(t)$. Thus, the velocity gradient tensor $\boldsymbol{\epsilon}(t)$ transforms into the streamline system as $\boldsymbol{\epsilon}^{\prime}(t)=\mathbf{Q}(t)+\tilde{\boldsymbol{\epsilon}}(t)$. A quick calculation reveals that the components of $\mathbf{Q}(t)$ are given by $Q_{12}(t)=-Q_{21}(t)=$ $\tilde{\epsilon}_{21}(t)$, where we use that $\frac{d \mathbf{v}(t)}{d t}=\boldsymbol{\epsilon}(t) \mathbf{v}(t)$. This gives for the velocity gradient in the streamline system the upper triangular form

$$
\boldsymbol{\epsilon}^{\prime}(t)=\left[\begin{array}{cc}
\tilde{\epsilon}_{11}(t) & \sigma(t) \\
0 & -\tilde{\epsilon}_{11}(t)
\end{array}\right],
$$

where we define the shear rate $\sigma(t)=\tilde{\epsilon}_{12}(t)+\tilde{\epsilon}_{21}(t)$ along the streamline. Note that $\tilde{\epsilon}_{11}(t)=d v_{s}[s(t)] / d s$ by definition. Furthermore, due to the incompressibility of $\mathbf{u}(\mathbf{x})$, $\tilde{\epsilon}_{22}(t)=-\tilde{\epsilon}_{11}(t)$. For simplicity of notation, in the following we drop the primes. The upper triangular form of $\boldsymbol{\epsilon}(t)$ as a direct result of the transformation into the streamline system permits explicit solution of (5) and reveals the dynamic origins of algebraic stretching.

Thus, we can formulate the evolution equation (5) of a material strip in streamline coordinates as

$$
\begin{aligned}
& d z_{1}(s)=\frac{d v_{s}(s)}{v_{s}(s)} z_{1}(s)+\frac{\sigma(s)}{v_{s}(s)} z_{2}(s) d s \\
& d z_{2}(s)=-\frac{d v_{s}(s)}{v_{s}(s)} z_{2}(s),
\end{aligned}
$$

where we used (2) to express $\mathbf{z}(t)=\mathbf{z}[s(t)]$ in terms of the distance along the streamline. The system (7) can be integrated to

$$
\begin{aligned}
& z_{1}(s)=\frac{v_{s}(s)}{v_{s}(0)}\left[z_{1}(0)+z_{2}(0) \int_{0}^{s} d s^{\prime} \sigma\left(s^{\prime}\right) \frac{v_{s}(0)^{2}}{v_{s}\left(s^{\prime}\right)^{3}}\right] \\
& z_{2}(s)=\frac{v_{s}(0)}{v_{s}(s)} z_{2}(0) .
\end{aligned}
$$

Note that the deformation tensor $\mathbf{F}(t)$ in the streamline system has also an upper triangular form. Its components can be directly read off the system (8). The angle of the strip $\mathbf{z}(t)$ with respect to the streamline orientation is denoted by $\phi(t)$ such that $z_{1}(t)=\ell(t) \cos [\phi(t)]$ and $z_{2}(t)=\ell(t) \sin [\phi(t)]$. The initial strip length and angle are denoted by $\ell_{0}$ and $\phi_{0}$. The strip length is given by $\ell(t) \equiv \ell[s(t)]$ with $\ell(s)=\left[z_{1}(s)^{2}+z_{2}(s)^{2}\right]^{1 / 2}$.

The system (8) is of general validity for $d=2$ dimensional steady flow fields. It reveals the mechanisms that lead to an increase of the strip elongation, which is fully determined by the shear deformation $\sigma(s)$ and the velocity $v_{s}(s)$ along the streamline. For a strip that is initially aligned with the streamline, $z_{2}(0)=0$, the elongation is $\ell(s)=z_{2}(0) v_{s}(s) / v_{s}(0)$ because $z_{2}(s) \equiv 0$ remains zero. This means $\ell(s)$ merely fluctuates without a net increase [53]. Only if the strip is oriented away from the streamline can the streamwise velocity fluctuations be converted into stretching. This identifies the integral term in (8a) as the dominant contribution to the strip 
a)

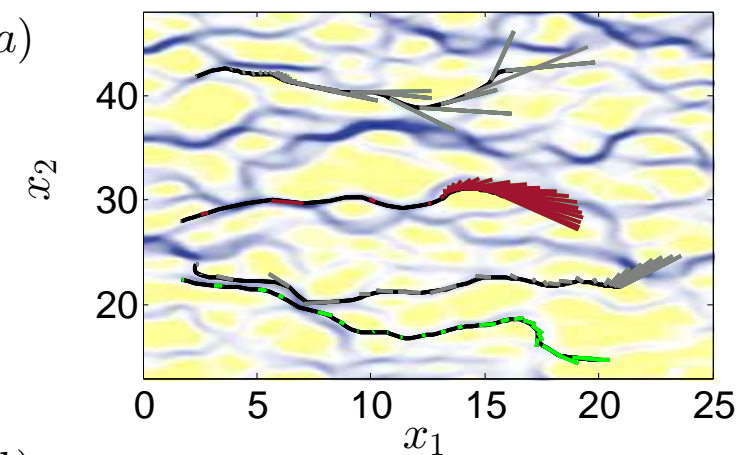

b)

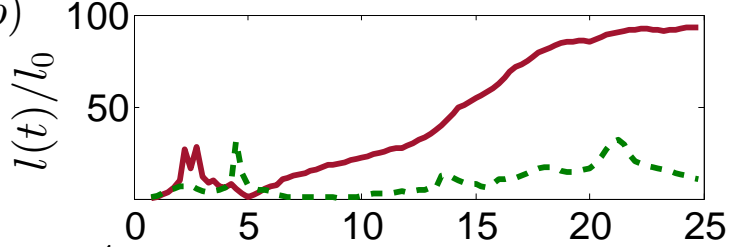

c)
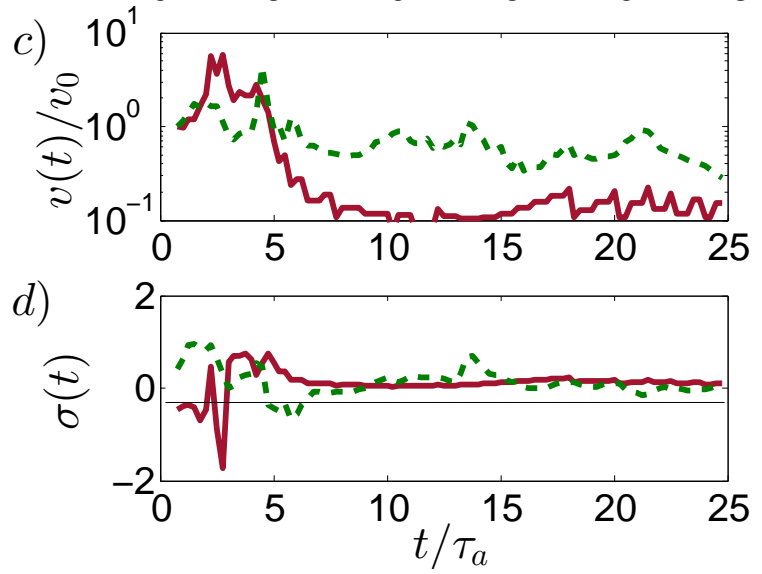

FIG. 1. (a) Illustration of the evolution of length (rescaled) and orientation of material strips along streamlines in a steady $d=2$ dimensional divergence-free random flow [19]. The color scales illustrate the velocity magnitude decreasing from blue to yellow. Strips are drawn along streamlines at equidistant times. We observe persistent stretching in low velocity zones. This is reflected in the bottom panels, which illustrates (b) strip elongations $\ell(t)$ for two distinct streamlines characterized by high (green dashed) and low (red solid) velocities, (c) strip velocity time series, and (d) shear deformation corresponding to the strip evolutions illustrated in the left panel by the same colors.

elongation. It represents the interaction of shear deformation and velocity with a linear contribution from the shear rate and a non-linear contribution from velocity as $1 / v_{s}(s)^{3}$, which may be understood as follows. One power comes from the divergence of streamlines in low velocity zones, which increases $z_{2}(s)$ and thus leads to enhanced shear deformation. The second power is purely kinematic due to the weighting with the residence time in a streamline segment. The third power stems from the fact that shear deformation in low velocity segments is applied while the strip is compressed in streamline direction. This deformation is then amplified as the strip is stretched due to velocity increase. As a result of this non-linear coupling, the history of low velocity episodes has a significant impact on the net stretching as quantified by the integral term in (8b). This persistent effect is superposed with the local velocity fluctuations. These mechanisms are illustrated in Figure 1. While for a stratified flow field with $\mathbf{u}(\mathbf{x})=\mathbf{u}\left(x_{2}\right)$ velocity and shear deformation are constant along a streamline such that $\ell(t)=\left[\left(z_{1}(0)+z_{2}(0) \sigma t\right)^{2}+z_{2}(0)^{2}\right]^{1 / 2}$, that is, it increases linearly with time, stretching can in general be sub- or superlinear, depending on the duration of low velocity episodes. In the following, we will analyze these behaviors in order to identify and quantify the origins of algebraic stretching.

\section{DEFORMATION CTRW}

To investigate the consequences of the non-linear coupling between shear and velocity on the emergence of sub-exponential stretching, we cast the dynamics (8) in the framework of a CTRW for the Lagrangian flow velocities $v_{s}(s)$ [48]. Thus, we assume that the random flow field is stationary and ergodic [54]. Furthermore, we assume Lagrangian ergodicity [55], this means fluid elements can sample the full velocity spectrum along a streamline [56]. As outlined in the Introduction, flow through heterogeneous porous media are in general characterized by open streamlines so that fluid particles can sample the full velocity spectrum as they move along a trajectory. This is not the case for flows in stratified media, in which velocities are perfectly correlated. Thus, here we consider random flows $\mathbf{u}(\mathbf{x})$ whose velocities fluctuations are controlled by a characteristic correlation length scale and focus on the impact of broad velocity point distributions rather than on that of long range correlation $[30,57]$. This is particularly relevant for porous media flows. It has been observed at the pore and Darcy scales that the streamwise velocity, that is, the velocity measured equidistantly along a streamline follows a Markov process [38, 40, 41, 46, 58]. Thus, if we choose a coarse-graining scale that is of the order of the streamwise correlation length $\lambda_{c},(2)$ can be discretized as

$$
s_{n+1}=s_{n}+\lambda_{c}, \quad t_{n+1}=t_{n}+\frac{\lambda_{c}}{v_{n}} .
$$

The $v_{n}=v_{s}\left(s_{n}\right)$ are identical independently distributed random velocities with the probability density function (PDF) $p_{v}(v)$. A result of this spatial Markovianity is that the particle movement follows a continuous time random walk (CTRW) $[32,51]$. The PDF of streamwise velocities $p_{v}(v)$ is related to the Eulerian velocity $\mathrm{PDF} p_{e}(v)$ through flux weighting as $p_{v}(v) \propto v p_{e}(v)$. The Eulerian velocity $\mathrm{PDF}$ in $d=2$ dimensional pore-networks, for example, can be approximated by a Gaussian-shaped distribution, which breaks down for small velocities [59]. For Darcy scale porous and fractured media the velocity PDF can be characterized by algebraic behaviors at 
small velocities $[32,41,60]$, which implies a broad distribution of transition times $\tau_{n}=\lambda_{c} / v_{n}$. Note, however, that the proposed CTRW stretching mechanisms is of general nature and valid for any velocity distribution $p_{v}(v)$. Thus, in order to extract the deformation dynamics, we coarse-grain the elongation process along the streamline on the correlation scale $\lambda_{c}$. This gives for the strip coordinates $(8)$

$$
\begin{aligned}
& z_{1}\left(s_{n}\right)=z_{1}(0) \frac{v_{n}}{v_{0}}+z_{2}(0) \frac{v_{n} v_{0}}{v_{c}^{2}} \sigma_{c} \tau_{v} r_{n} \\
& z_{2}\left(s_{n}\right)=z_{2}(0) \frac{v_{0}}{v_{n}}
\end{aligned}
$$

with $v_{c}$ and $\sigma_{c}$ a characteristic velocity and shear rate, and $\tau_{v}=\lambda_{c} / v_{c}$ a characteristic advection time. The process $r_{n}$, which results from the integral term in (8a), describes the coupled CTRW

$$
r_{n+1}=r_{n}+\frac{v_{c}^{3}}{\sigma_{c}} \frac{\sigma_{n}}{v_{n}^{3}}, \quad t_{n+1}=t_{n}+\frac{\lambda_{c}}{v_{n}} .
$$

The elongation at time $t$ is given by $\ell(t)=\left[z_{1}\left(s_{n_{t}}\right)^{2}+\right.$ $\left.z_{2}\left(s_{n_{t}}\right)^{2}\right]^{1 / 2}$. It is observed over several $2 d$ flows that the shear rate may be related to the streamwise velocity as $\sigma_{n}=\xi_{n} \sigma_{c}\left(v_{n} / v_{c}\right)^{\hat{\alpha}}$ with $\hat{\alpha} \approx 1, \sigma_{c}$ a characteristic shear rate, and $\xi_{n}$ an identical independent random variable that is equal to \pm 1 with equal probability. The average shear rate $\left\langle\sigma_{n}\right\rangle=0$ due to the stationarity of the random flow field $\mathbf{u}(\mathbf{x})$. Thus, (11) denotes a coupled CTRW whose increments $\rho_{n} \equiv r_{n+1}-r_{n}$ are related to the transition times $\tau_{n}=\lambda_{c} / v_{n}$ as

$$
\rho_{n}=\xi_{n}\left(\tau_{n} / \tau_{v}\right)^{\alpha}, \quad \alpha=3-\hat{\alpha} .
$$

It has the average $\left\langle\rho_{n}\right\rangle=0$ and absolute value $\left|\rho_{n}\right|=$ $\left(\tau_{n} / \tau_{v}\right)^{\alpha}$. The joint PDF of the elongation increments $\rho$ and transition times $\tau$ is then given by

$$
\psi(\rho, \tau)=\frac{1}{2} \delta\left[|\rho|-\left(\tau / \tau_{v}\right)^{\alpha}\right] \psi(\tau),
$$

where $\delta(\rho)$ denotes the Dirac delta distribution. The transition time $\operatorname{PDF} \psi(\tau)$ is related to the streamwise velocity $\operatorname{PDF} p_{v}(v)$ as $\psi(\tau)=\lambda_{c} \tau^{-2} p_{v}\left(\lambda_{c} / \tau\right)$.

\section{ALGEBRAIC STRETCHING}

In the following, we consider a streamwise velocity PDF that behaves as $p_{v}(v) \propto\left(v / v_{c}\right)^{\beta-1}$ for $v$ smaller than the characteristic velocity $v_{c}$. Such a power-law is a model for the low end of the velocity spectra in disordered media [30] and porous media flows [36, 39, 41]. Note however that the derived CTRW-based deformation mechanism is valid for any velocity distribution. The relation between the streamwise and Eulerian velocity PDFs, $p_{v}(v) \propto v p_{e}(v)$ implies that $\beta \geq 1$ because $p_{e}(v)$ needs to be integrable in $v=0$. The corresponding transition time $\operatorname{PDF} \psi(\tau)$ behaves as $\psi(\tau) \propto\left(\tau / \tau_{v}\right)^{-1-\beta}$ for

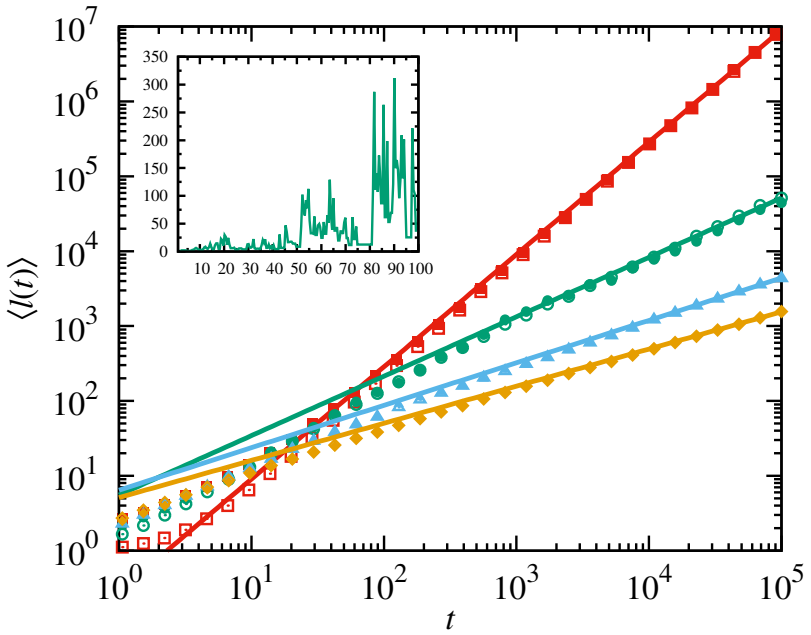

FIG. 2. Evolution of the (open symbols) mean elongation $\langle\ell(t)\rangle=\left\langle\left[z_{1}\left(s_{n_{t}}\right)^{2}+z_{2}\left(s_{n_{t}}\right)^{2}\right]^{1 / 2}\right\rangle$ with $\ell_{n}$ given obtained from numerical Monte-Carlo simulations using (10) and (11) for a uniform distribution of initial strip orientations $\phi \in[-\pi / 2, \pi / 2]$ and a Gamma PDF of streamwise velocity. The full symbols are obtained from the approximation (14) for (squares) $\beta=3 / 2$, (circles) $\beta=5 / 2$, (triangles) $\beta=7 / 2$ and (rhombi) $\beta=9 / 2$. The solid lines indicate the late time power-law behaviors of $\langle\ell(t)\rangle \propto t^{3-\beta}$ for $1<\beta<2$, $\langle\ell(t)\rangle \propto t^{2 / \beta}$ for $2<\beta<4$, and $\langle\ell(t)\rangle \propto t^{1 / 2}$ for $\beta>4$. The inset illustrates the evolution of $\ell(t)$ in a single realization of the velocity field $v_{n}$ for $\beta=5 / 2$ for an initial orientation of $\phi=0$.

$\tau>\tau_{v}=\lambda_{c} / v$ and decreases sharply for $\tau<\tau_{v}$. Due to the constraint $\beta>1$, the mean transition time $\langle\tau\rangle<\infty$ is always finite, which is a consequence of fluid mass conservation. For transport in highly heterogeneous pore Darcy-scale porous media values for $\beta$ between 0 and 2 have been reported $[36,39]$. It has been found that decreasing medium heterogeneity leads to a sharpening of the transition time PDF and increase of the exponent $\beta$ [41] with $\beta>1$. With these definitions, the coupled CTRW (11) describes a Levy walk.

Figure 2 shows the evolution of the average elongation $\langle\ell(t)\rangle$ for $\alpha=0$ and different values of $\beta$ obtained from numerical Monte-Carlo simulation using (10) and the Levy walk (11) for the evolution of the strip coordinates based on a Gamma PDF of streamwise velocities [53]. The mean elongation shows a power-law behavior and increases as $\langle\ell(t)\rangle \propto t^{\nu}$. As discussed above, long episodes of small velocity maintain the strip in a favorable shear angle, which leads to a strong stretching. These dynamics are quantified by the Lévy walk process (11), which relates strong elongations to long transition times, i.e., small streamwise velocities, through (12). This is also illustrated in the inset of Figure 2, which shows the elongation of a single material strip. The elongation events increase with increasing time as a consequence of the coupling (12) between stretching and transition time. This is 
an intrinsic property of a CTRW characterized by a broad $\psi(\tau)$; the transition times increase as time increases, and thus, through the Levy walk coupling also the stretching increments. In fact, the strip length can be approximated by $[53]$

$$
\ell(t) \approx \ell_{0}+\frac{\sigma_{c} \tau_{v}^{2}\langle v\rangle}{\langle\tau\rangle v_{c}}\left|z_{2}(0)\right|\left|r_{n_{t}}\right|
$$

The leading behavior of the mean elongation $\langle\ell(t)\rangle$ of a material element is directly related to the mean absolute moment of $r(t)$ as $\langle\ell(t)\rangle \propto\left\langle\left|r_{n_{t}}\right|\right\rangle$. Thus, even though $r_{n_{t}}$ is in average 0 , the addition of large elongation events in its absolute value $|r(t)|$, which correspond to episodes of low velocities, leads in average to an algebraic increase of $\ell(t)$ as detailed in the following.

The statistics of the Levy walk (11) have been analyzed in detail in Ref. [61] for $\alpha>0$ and $\beta>0$. Here, $\beta$ is restricted to $\beta>1$ due to fluid mass conservation. Furthermore, we consider $\alpha \geq 1$. The scaling of the mean absolute moments of $r_{n_{t}}$ depends on the $\alpha$ and $\beta$ regimes.

If the exponent $\beta>2 \alpha$, which means a relatively weak heterogeneity, we speak of a weak coupling between the elongation increment $\rho_{n}$ and the transition time $\tau_{n}$ in (12). In this case, the strip elongation behaves as $\langle\ell(t)\rangle \propto t^{1 / 2}$. We term this behavior here diffusive or normal stretching. For $\alpha=2$ as employed in the numerical simulations this means that $\beta>4$. The coupled Levy-walk (11) reduces essentially to a Brownian motion because the variability of transition times is low so that the coupling does not lead to strong elongation events. Note that scalar dispersion in this $\beta$-range is normal [36, 51].

For strong coupling, this means $\beta<2 \alpha$ and thus stronger flow heterogeneity, it has been shown [61] that the density of $r_{n_{t}}$ is characterized by two scaling forms, one that characterizes the bulk behavior and a different one for large $r_{n_{t}}$. As a consequence, we need to distinguish the cases of $\beta$ larger and smaller than $\alpha$. Also, the scaling of $\left|r_{n_{t}}\right|$ cannot be obtained by dimensional analysis. In fact, $r_{n_{t}}$ has a strong anomalous diffusive character [61].

For $\alpha<\beta<2 \alpha$ the scaling behavior of the mean elongation is $\langle\ell(t)\rangle \propto t^{\alpha / \beta}$. This means for $\alpha=2$, the stretching exponent $\nu$ is between $1 / 2$ and 1 , the $\beta$-range is $2<\beta<4$. It interesting to note that scalar dispersion in this range is normal as well. Here, the frequency of low velocity regions is high enough to increase stretching above the weakly coupled case, but not to cause superdiffusive scalar dispersion.

For $1<\beta<\alpha$ in contrast, the mean elongation scales as $[61]\langle\ell(t)\rangle \propto t^{1+\alpha-\beta}$. The stretching exponent is between 1 and $\alpha$, this means stretching is stronger than for shear flow. The range of scaling exponents $\nu$ of the mean elongation here is $1 / 2 \leq \nu<\alpha$. Specifically, $\alpha \approx 2$ implies that stretching is super-linear for $1<\beta<2$, this means faster than by a pure shear flow, for which $\nu=1$. Here the presence of low velocities in the flow leads to enhanced stretching and at the same time to superdiffusive scalar dispersion.

\section{SUMMARY AND CONCLUSIONS}

In summary, we have presented a fundamental mechanism for power-law stretching in random flows through intermittent shear events, which may explain algebraic mixing processes observed across a range of heterogeneous flows. We have shown that the non-linear coupling between streamwise velocities and shear deformation implies that stretching follows a coupled CTRW, which explains observed subexponential stretching behaviors that can range from diffusive to super-diffusive scalings, $\langle\ell(t)\rangle \propto t^{\nu}$ with $1 / 2 \leq \nu<2$. The derived coupled stretching CTRW can be parameterized in terms of the Eulerian velocity and deformation statistics and provides a link between anomalous dispersion and fluid deformation. The presented analysis demonstrates that the dynamics of fluid stretching in heterogeneous flow fields is much richer than the paradigmatic linear and exponential behaviors. The fundamental mechanism of intermittent shear events, which is at the root of nonexponential stretching, is likely present in a broader class of fluid flows.

\section{ACKNOWLEDGMENTS}

MD acknowledges the support of the European Research Council (ERC) through the project MHetScale (contract number 617511). TLB acknowledges Agence National de Recherche (ANR) funding through the project ANR-14-CE04-0003-01.
[1] M. F. Shlesinger, B. J. West, and J. Klafter, Phys. Rev. Lett. 58, 1100 (1987).

[2] M. P. Rast and J.-F. Pinton, Phys. Rev. Lett. 107, 214501 (2011).

[3] S. Thalabard, G. Krstulovic, and J. Bec, J. Fluid Mech. 755, R4 (2014).
[4] N. Kleinfelter, M. Moroni, and J. H. Cushman, Phys. Rev. E 72, 056306 (2005).

[5] E. Villermaux and J. Duplat, Phys. Rev. Lett. 97, 144506 (2006).

[6] B. Jha, L. Cueto-Felgueroso, and R. Juanes, Phys. Rev. Lett. 106, 194502 (2011). 
[7] F. P. J. De Barros, M. Dentz, J. Koch, and W. Nowak, Geophys. Res. Lett. 39, L08404 (2012).

[8] T. Le Borgne, M. Dentz, and E. Villermaux, J. Fluid Mech. 770, 458 (2015).

[9] Y. Ye, G. Chiogna, O. A. Cirpka, P. Gratwohl, and M. Rolle, Phys. Rev. Lett. 115, 194502 (2015).

[10] W. E. Ranz, AIChE Journal 25, 41 (1979).

[11] A. M. Tartakovsky, D. M. Tartakovsky, and P. Meakin, Phys. Rev. Lett. 101, 044502 (2008).

[12] N. B. Engdahl, D. A. Benson, and D. Bolster, Phys. Rev. E 90, 051001(R) (2014).

[13] J. J. Hidalgo, M. Dentz, Y. Cabeza, and J. Carrera, Geophys. Res. Lett. 42, 6375 (2015).

[14] G. Lapeyre, P. Klein, and P. L. Hua, Phys. Fluids 11, 3729 (1999).

[15] C. Truesdell and W. Noll, The Non-Linear Field Theories of Mechanics (Springer-Verlag, 1992).

[16] J. Kalda, Phys. Rev. Letters 84, 471 (2000).

[17] E. Villermaux and J. Duplat, Phys. Rev. Lett. 91, 18 (2003).

[18] E. Villermaux, C. R. Mécanique 340, 933 (2012).

[19] T. Le Borgne, M. Dentz, and E. Villermaux, Phys. Rev. Lett. 110, 204501 (2013).

[20] J. Ottino, The Kinematics of Mixing: Stretching, Chaos, and Transport (Cambridge University Press, 1989).

[21] J. Duplat and E. Villermaux, Eur. Phys. J. B 18, 353 (2000).

[22] J. Duplat, C. Innocenti, and E. Villermaux, Phys. Fluids 22, 035104 (2010).

[23] S. Goto and J. C. Vassilicos, New J. Phys. 6, 1 (2004).

[24] E. Afik and V. Steinberg, arXiv:1502.02818 (2015).

[25] M. Nelkin and R. M. Kerr, Phys. Fluids 24, 9 (1981).

[26] J. C. Vassilicos, Phil. Trans. R. Soc. Lond. 360, 2819 (2002).

[27] G. Bofetta and I. M. Sokolov, Phys. Rev. Lett. 88, 094501 (2002).

[28] J. Bear, Dynamics of Fuids in Porous Media (American Elsevier, New York, 1972).

[29] H. Brenner and D. A. Edwards, Macrotransport Processes (Butterworth-Heinemann, 1993).

[30] J. P. Bouchaud and A. Georges, Phys. Rep. 195, 127 (1990).

[31] J. H. Cushman, X. Hu, and T. R. Ginn, J. Stat. Phys. 75, 859 (1994).

[32] B. Berkowitz and H. Scher, Phys. Rev. Lett. 79, 4038 (1997).

[33] D. A. Benson, S. W. Wheatcrat, and M. M. Meerschaert, Water Resour. Res. 36, 1403? (2000).

[34] R. Haggerty, S. A. McKenna, and L. C. Meigs, Water Resour. Res. 36, 3467 (2000).

[35] J. D. Seymour, J. P. Gage, S. L. Codd, and R. Gerlach, Phys. Rev. Lett. 93, 198103 (2004).

[36] B. Berkowitz, A. Cortis, M. Dentz, and H. Scher, Rev. Geophys. 44, 2005RG000178 (2006).

[37] S. P. Neuman and D. M. Tartakovsky, Adv. Water Resour. 32, 670 (2008).
[38] T. Le Borgne, M. Dentz, and J. Carrera, Phys. Rev. Lett. 101, 090601 (2008).

[39] B. Bijeljic, P. Mostaghimi, and M. Blunt, Phys. Rev. Lett. 107, 204502 (2011).

[40] P. De Anna, T. Le Borgne, M. Dentz, A. Tartakovsky, D. Bolster, and P. Davy, Phys. Rev. Lett. 110, 184502 (2013).

[41] Y. Edery, A. Guadagnini, H. Scher, and B. Berkowitz, Water Resour. Res. 50, doi:10.1002/2013WR015111 (2014).

[42] A. Tyukhova, D. M., W. Kinzelbach, and M. Willmann, Phys. Rev. Fluids 1, 074002 (2016).

[43] G. Sposito, Water Resour. Res. 30, 2395 (1994).

[44] G. Sposito, Adv. Water Resour. 24, 793 (2001).

[45] M. Dentz, H. Kinzelbach, S. Attinger, and W. Kinzelbach, Physical Review E 67, 046306 (2005).

[46] T. Le Borgne, M. Dentz, and J. Carrera, Phys. Rev. E 78, 026308 (2008).

[47] V. Cvetkovic, A. Fiori, and G. Dagan, Water Resources Research 50, 5759 (2014), cited By 6.

[48] M. Dentz, P. K. Kang, A. Comolli, T. Le Borgne, and D. R. Lester, Phys. Rev. Fluids 1, 074004 (2016).

[49] H. Winter, Journal of Non-Newtonian Fluid Mechanics 10, 157 (1982).

[50] E. W. Montroll and G. H. Weiss, J. Math. Phys. 6, 167 (1965).

[51] H. Scher and M. Lax, Phys. Rev. B 7, 4491 (1973).

[52] A. Zaburdaev, S. Denisov, and J. Klafter, Rev. Mod. Phys. 87, 483 (2015).

[53] See Supplemental Material at [URL will be inserted by publisher] for details on the derivation of the equations for the strip deformation in the streamline coordinate system, the approximations for the calculation of the average strip elongation, and the numerical random walk particle tracking simulations.

[54] G. Christakos, Random Field Models in Earth Sciences (Academic Press, 1992).

[55] A. M. Yaglom, An Introduction to the Theory of Stationary Random Functions (Dover Publications, 1962).

[56] For random flows displaying open and closed streamlines such as the steady $d=2$ dimensional Kraichnan model, we focus on stretching in the subset of ergodic streamlines. Stretching due to shear on closed streamlines is linear in time.

[57] M. Dentz and D. Bolster, Phys. Rev. Lett. 105, 244301 (2010).

[58] P. K. Kang, M. Dentz, T. Le Borgne, and R. Juanes, Phys. Rev. Lett. 107, 180602, doi:10.1103/PhysRevLett.107.180602 (2011).

[59] A. D. Araújo, B. B. Wagner, J. S. Andrade, and H. Herrmann, Phys. Rev. E 74, 010401(R) (2006).

[60] P. K. Kang, D. M., L. B. T., and R. Juanes, Phys. Rev. E 92, 022148 (2015).

[61] M. Dentz, T. Le Borgne, D. R. Lester, and F. P. J. de Barros, Phys. Rev. E 92, 032128 (2015). 


\title{
Supplemental Material: Coupled continuous time random walks for fluid stretching in two-dimensional heterogeneous media
}

\author{
Marco Dentz \\ Spanish National Research Council (IDAEA-CSIC), 08034 Barcelona, Spain* \\ Daniel R. Lester \\ School of Civil, Environmental and Chemical Engineering, \\ RMIT University, 3000 Melbourne, Victoria, Australia \\ Tanguy Le Borgne \\ Geosciences Rennes, UMR 6118, Université de Rennes 1, CNRS, Rennes, France
}

Felipe P. J. de Barros

Sonny Astani Department of Civil and Environmental Engineering, University of Southern California, 3620 S. Vermont Avenue, KAP 224B, Los Angeles, CA 90089, USA

(Dated: November 30, 2016)

\begin{abstract}
This supplementary material gives details on the derivation of the equations for the strip deformation in the streamline coordinate system, the approximations for the calculation of the average strip elongation, and the numerical random walk particle tracking simulations.
\end{abstract}

\section{DEFORMATION IN THE STREAMLINE COORDINATE SYSTEM}

Here we briefly present the key steps leading to the explicit solutions for the evolution of a material strip in the streamline coordinate system. First, we note that the deformation rate tensor $\boldsymbol{\epsilon}^{\prime}(t)$ in the streamline coordinate system is triangular. This can be seen as follows. As derived in the main text, $\boldsymbol{\epsilon}^{\prime}(t)$ is given by

$$
\boldsymbol{\epsilon}^{\prime}(t)=\mathbf{Q}(t)+\tilde{\epsilon}(t)
$$

where $\mathbf{Q}(t)=d \mathbf{A}^{\top}(t) / d t \mathbf{A}(t)$ and $\tilde{\epsilon}(t)=\mathbf{A}^{\top}(t) \boldsymbol{\epsilon}(t) \mathbf{A}(t)$. Note that $\mathbf{A}(t)=\left[\mathbf{e}_{v}(t), \mathbf{e}_{w}\right]$, with $\mathbf{e}_{v}(t)=\mathbf{v}(t) / v(t)$ and $\mathbf{e}_{w}(t)=\mathbf{w}(t) / v(t)$ and $\mathbf{v}(t) \cdot \mathbf{w}(t)=0$. Thus, we obtain directly that $Q_{11}(t)=Q_{22}(t)=0$ and $Q_{12}(t)=-Q_{21}(t)=$ $\mathbf{e}_{w}(t) d \mathbf{e}_{v}(t) / d t$, where

$$
\begin{aligned}
\frac{d \mathbf{e}_{v}(t)}{d t} & =-\frac{d \ln v(t)}{d t} \mathbf{e}_{v}(t)+\frac{1}{v(t)} \frac{d \mathbf{v}(t)}{d t}=-\frac{d \ln v(t)}{d t} \mathbf{e}_{v}(t)+\frac{1}{v(t)} \boldsymbol{\epsilon}(t) \mathbf{v}(t) \\
& =-\frac{d \ln v(t)}{d t} \mathbf{e}_{v}(t)+\boldsymbol{\epsilon}(t) \mathbf{e}_{v}(t)
\end{aligned}
$$

From the latter we obtain directly $Q_{12}(t)=\tilde{\epsilon}_{21}(t)$, which gives the triangluar form of $\boldsymbol{\epsilon}^{\prime}(t)$.

The equations for the strip components $z_{1}^{\prime}(t)$ and $z_{2}^{\prime}(t)$ then are given by

$$
\begin{aligned}
& \frac{d z_{1}^{\prime}(t)}{d t}=\epsilon_{11}^{\prime}(t) z_{1}^{\prime}(t)+\sigma(t) z_{2}^{\prime}(t) \\
& \frac{d z_{2}^{\prime}(t)}{d t}=-\epsilon_{11}^{\prime}(t) z_{2}^{\prime}(t) .
\end{aligned}
$$

We transform now $d t=d s / v_{s}(s)$ and note that $\epsilon_{11}^{\prime}=d v_{s} / d s$ in order to obtain

$$
\begin{aligned}
& \frac{d z_{1}^{\prime}(s)}{d s}=v_{s}(s)^{-1} \frac{d v_{s}(s)}{d s} z_{1}^{\prime}(s)+\frac{\sigma(s)}{v_{s}(s)} z_{2}^{\prime}(s) \\
& \frac{d z_{2}^{\prime}(s)}{d s}=-v_{s}(s)^{-1} \frac{d v_{s}(s)}{d s} z_{2}^{\prime}(s) .
\end{aligned}
$$

\footnotetext{
* marco.dentz@csic.es
} 
This system can be integrated. We start with the second equation for $z_{2}^{\prime}(s)$, which can be written as

$$
\frac{d z_{2}^{\prime}(s)}{z_{2}^{\prime}(s)}=-\frac{d v_{s}(s)}{v_{s}(s)}
$$

Thus, it can be directly integrated to

$$
\ln \left[z_{2}^{\prime}(s) / z_{2}^{\prime}(0)\right]=-\ln \left[v_{s}(s) / v_{s}(0)\right]
$$

Taking the natural exponential on both sides gives

$$
z_{2}^{\prime}(s)=\frac{v_{s}(0)}{v_{s}(s)} z_{2}^{\prime}(0)
$$

Inserting the latter into (6), we obtain

$$
\frac{d z_{1}^{\prime}(s)}{d s}=v_{s}(s)^{-1} \frac{d v_{s}(s)}{d s} z_{1}^{\prime}(s)+\frac{\sigma(s) v_{s}(0)}{v_{s}(s)^{2}} z_{2}^{\prime}(0) .
$$

This equation can be solved by separation of variables. Thus, we write $z_{1}^{\prime}(s)=f(s) g(s)$, where $f(s)$ satifies

$$
\frac{d f(s)}{d s}=v_{s}(s)^{-1} \frac{d v_{s}(s)}{d s} f(s)
$$

Its solution is

$$
f(s)=f(0) \frac{v_{s}(s)}{v_{s}(0)}
$$

By inserting $z_{1}^{\prime}(s)=f(0) v_{s}(s) / v_{s}(0) g(s)$ into (11) gives for $g(s)$ the equation

$$
\frac{d g(s)}{d s}=\frac{1}{f(s)} \frac{\sigma(s) v_{s}(0)}{v_{s}(s)^{2}} z_{2}^{\prime}(0)=f(0)^{-1} \frac{\sigma(s) v_{s}(0)^{2}}{v_{s}(s)^{3}} z_{2}^{\prime}(0)
$$

Integration of the latter yields

$$
g(s)=g(0)+f(0)^{-1} z_{2}^{\prime}(0) \int_{0}^{s} d s^{\prime} \frac{\sigma\left(s^{\prime}\right) v_{s}(0)^{2}}{v_{s}\left(s^{\prime}\right)^{3}}
$$

and thus for $z_{1}^{\prime}(s)$

$$
z_{1}^{\prime}(s)=g(0) f(0) \frac{v_{s}(s)}{v_{s}(0)}+z_{2}^{\prime}(0) \frac{v_{s}(s)}{v_{s}(0)} \int_{0}^{s} d s^{\prime} \frac{\sigma\left(s^{\prime}\right) v_{s}(0)^{2}}{v_{s}\left(s^{\prime}\right)^{3}}
$$

The integration constants $g(0) f(0)$ are determined by the initial condition $z_{1}^{\prime}(0)$ such that

$$
z_{1}^{\prime}(s)=z_{1}^{\prime}(0) \frac{v_{s}(s)}{v_{s}(0)}+z_{2}^{\prime}(0) \frac{v_{s}(s)}{v_{s}(0)} \int_{0}^{s} d s^{\prime} \frac{\sigma\left(s^{\prime}\right) v_{s}(0)^{2}}{v_{s}\left(s^{\prime}\right)^{3}} .
$$

\section{STRIP ELONGATION}

In order to derive Eq. (14) in the main text for the strip elongation, we use the fact that $v_{n_{t}} / v_{0}$ in (10a) in the main text evolves in average towards 1 , and that $v_{0} / v_{n_{t}}$ in $(10 \mathrm{~b})$ in the main text evolves in average slower than $\left|r_{n_{t}}\right|$ as detailed below. Thus, we disregard $v_{n_{t}} / v_{0}$ and $v_{0} / v_{n_{t}}$ as subleading. Furthermore, as shown below, we use that $v_{s}(s) v_{s}(0)$ converges in average towards the constant $\langle v\rangle \lambda_{c} /\langle\tau\rangle$ in order to obtain expression (14) in the main text. 


\section{A. Average Elongation Perpendicular to the Streamline}

We first determine the average deformation of the material strip perpendicular to the streamline, $\left\langle\left|z_{2}(t)\right|\right\rangle$. It is given by

$$
\left\langle\left|z_{2}(t)\right|\right\rangle=\left\langle\left|z_{2}(0)\right|\right\rangle\left\langle\frac{v_{0}}{v_{n_{t}}}\right\rangle=\left\langle z_{2}(0)\right\rangle \sum_{n=0}^{\infty}\left\langle\frac{v_{0}}{v_{n}} \int_{0}^{t} d t^{\prime} \delta\left(t^{\prime}-t_{n}\right) \mathbb{I}\left(0 \leq t-t^{\prime}<\tau_{n}\right)\right\rangle .
$$

It reads in Laplace space as

$$
\begin{aligned}
\frac{\left\langle\left|z_{2}(\lambda)\right|\right\rangle}{\left\langle\left|z_{2}(0)\right|\right\rangle} & =\sum_{n=0}^{\infty}\left\langle\frac{v_{0}}{v_{n}} \exp \left(-\lambda t_{n}\right) \frac{1-\exp \left(-\lambda \tau_{n}\right)}{\lambda}\right\rangle \\
& =\frac{1-\psi(\lambda)}{\lambda}+\left\langle v_{0} \exp \left(-\lambda \tau_{0}\right)\right\rangle \sum_{n=1}^{\infty} \psi(\lambda)^{n-1}\left\langle\frac{1}{v_{n}} \frac{1-\exp \left(-\lambda \tau_{n}\right)}{\lambda}\right\rangle \\
& =\frac{1-\psi(\lambda)}{\lambda}+\frac{\left\langle\tau_{0}^{-1} \exp \left(-\lambda \tau_{0}\right)\right\rangle}{1-\psi(\lambda)}\left\langle\tau \frac{1-\exp (-\lambda \tau)}{\lambda}\right\rangle \\
& =\frac{1-\psi(\lambda)}{\lambda}+\frac{1}{\lambda} \frac{\left\langle\tau_{0}^{-1} \exp \left(-\lambda \tau_{0}\right)\right\rangle}{1-\psi(\lambda)}\left[\langle\tau\rangle+\frac{d \psi(\lambda)}{d \lambda}\right]
\end{aligned}
$$

If $\left\langle\tau^{2}\right\rangle<\infty$, we obtain asymptotically

$$
\left\langle\left|z_{2}(\lambda)\right|\right\rangle \approx \frac{\left\langle\left|z_{2}(0)\right|\right\rangle\left\langle v_{0}\right\rangle\left\langle\tau^{2}\right\rangle}{\lambda s_{0}\langle\tau\rangle}
$$

Thus, the long time value is given by

$$
\lim _{t \rightarrow \infty}\left\langle\left|z_{2}(t)\right|\right\rangle \approx \frac{\left\langle\left|z_{2}(0)\right|\right\rangle\left\langle v_{0}\right\rangle\left\langle\tau^{2}\right\rangle}{s_{0}\langle\tau\rangle}
$$

In this Letter, we employ the Gamma distribution of streamwise velocities

$$
p_{v}(v)=\frac{\left(v / v_{c}\right)^{\beta-1} \exp \left(-v / v_{c}\right)}{v_{c} \Gamma(\beta)}
$$

The velocity moments are given by

$$
\left\langle v^{n}\right\rangle=\frac{v_{c}^{n} \Gamma(\beta+n)}{\Gamma(\beta)} .
$$

The moments of the transition times $\tau=s_{0} / v$ are given by

$$
\left\langle\tau^{n}\right\rangle=\frac{\tau_{v}^{n} \Gamma(\beta-n)}{\Gamma(\beta)} .
$$

for $\beta>2$.

Thus, for the gamma velocity PDF employed in this Letter, we obtain for the long time value

$$
\lim _{t \rightarrow \infty}\left\langle\left|z_{2}(t)\right|\right\rangle=\frac{\left\langle\left|z_{2}(0)\right|\right\rangle \Gamma(\beta+1) \Gamma(\beta-2)}{\Gamma(\beta) \Gamma(\beta-1)} .
$$

For $1<\beta<2$, the second moment $\left\langle\tau^{2}\right\rangle$ is not finite. In this case, we obtain in the limit $\lambda \tau_{v} \ll 1$ for the Laplace transform of $\psi(\tau)$

$$
\psi(\lambda)=1-\langle\tau\rangle \lambda+a_{\beta}\left(\lambda \tau_{v}\right)^{\beta}
$$

Thus, in the limit of $\lambda \tau_{v} \ll 1$ and $1<\beta<2$ we obtain for $\left\langle\left|z_{2}(\lambda)\right|\right\rangle$ in leading order

$$
\left\langle\left|z_{2}(\lambda)\right|\right\rangle \approx \frac{\left\langle\left|z_{2}(0)\right|\right\rangle\left\langle v_{0}\right\rangle}{s_{0} \lambda^{2}\langle\tau\rangle}\left[\langle\tau\rangle+\frac{d \psi(\lambda)}{d \lambda}\right] \propto \lambda^{\beta-3},
$$

which gives in real time the behavior

$$
\left\langle\left|z_{2}(t)\right|\right\rangle \propto t^{2-\beta}
$$



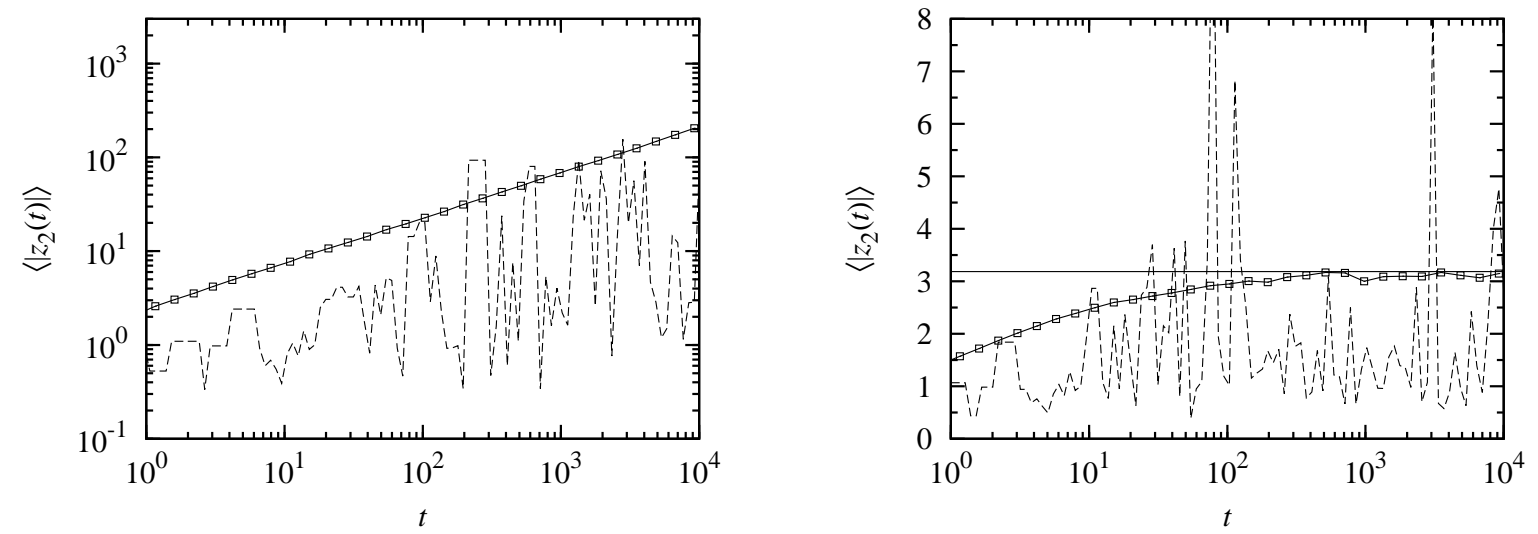

FIG. 1. (Symbols) Average elongation perpendicular to the streamline for (left panel) $\beta=3 / 2$ and (right panel) $\beta=5 / 2$. (Dashed) Evolution of elongation in a single realization for $z_{1}(0)=0$ and $z_{2}(0)=1$. The horizontal solid line in the right panel indicate the asymptotic value (28).

\section{B. Average Elongation Along the Streamline}

Now we consider the contribution $\delta z_{1}(t)=v_{n_{t}} / v_{0}$, which quantifies the elongation for a material strip that is initially aligned with the streamline, that is $z_{2}(0)=0$. Similarly as for $\left\langle z_{2}(t)\right\rangle$, we obtain for the Laplace transform of $\left\langle\delta z_{1}(t)\right\rangle$

$$
\begin{aligned}
\frac{\left\langle\left|\delta z_{1}(\lambda)\right|\right\rangle}{\left\langle\left|z_{1}(0)\right|\right\rangle} & =\frac{1-\psi(\lambda)}{\lambda}+\frac{\left\langle\tau_{0} \exp \left(-\lambda \tau_{0}\right)\right\rangle}{1-\psi(\lambda)}\left\langle\frac{1-\exp (-\lambda \tau)}{\lambda \tau}\right\rangle \\
& =\frac{1-\psi(\lambda)}{\lambda}+\frac{1}{\lambda} \frac{\left\langle\tau_{0} \exp \left(-\lambda \tau_{0}\right)\right\rangle}{1-\psi(\lambda)}\left[\frac{\langle v\rangle}{s_{0}}-\left\langle\tau^{-1} \exp (-\lambda \tau)\right\rangle\right] \\
& =\frac{1-\psi(\lambda)}{\lambda}-\frac{1}{\lambda[1-\psi(\lambda)]} \frac{d \psi_{0}(\lambda)}{d \lambda}\left[\frac{\langle v\rangle}{s_{0}}-\left\langle\tau^{-1} \exp (-\lambda \tau)\right\rangle\right]
\end{aligned}
$$

In the limit of $\lambda \ll 1$ and $1<\beta<2$ we obtain in leading order

$$
\left\langle\delta z_{1}(\lambda)\right\rangle \approx \frac{\left\langle\left|z_{1}(0)\right|\right\rangle}{\lambda}
$$

Thus, we obtain asymptotically

$$
\lim _{t \rightarrow \infty}\left\langle\delta z_{1}(t)\right\rangle=\left\langle\left|z_{1}(0)\right|\right\rangle
$$

\section{Average Velocity Cross-Product Along the Streamline}

Here, we determine the average velocity cross-product $C_{v}(t)=\langle v(0) v(t)\rangle$, which can be written as

$$
C_{v}(t)=\left\langle v_{0} v_{n_{t}}\right\rangle=\sum_{n=0}^{\infty}\left\langle v_{0} v_{n} \int_{0}^{t} \delta\left(t^{\prime}-t_{n}\right) \mathbb{I}\left(0 \leq t-t^{\prime}<\tau_{n}\right)\right\rangle
$$



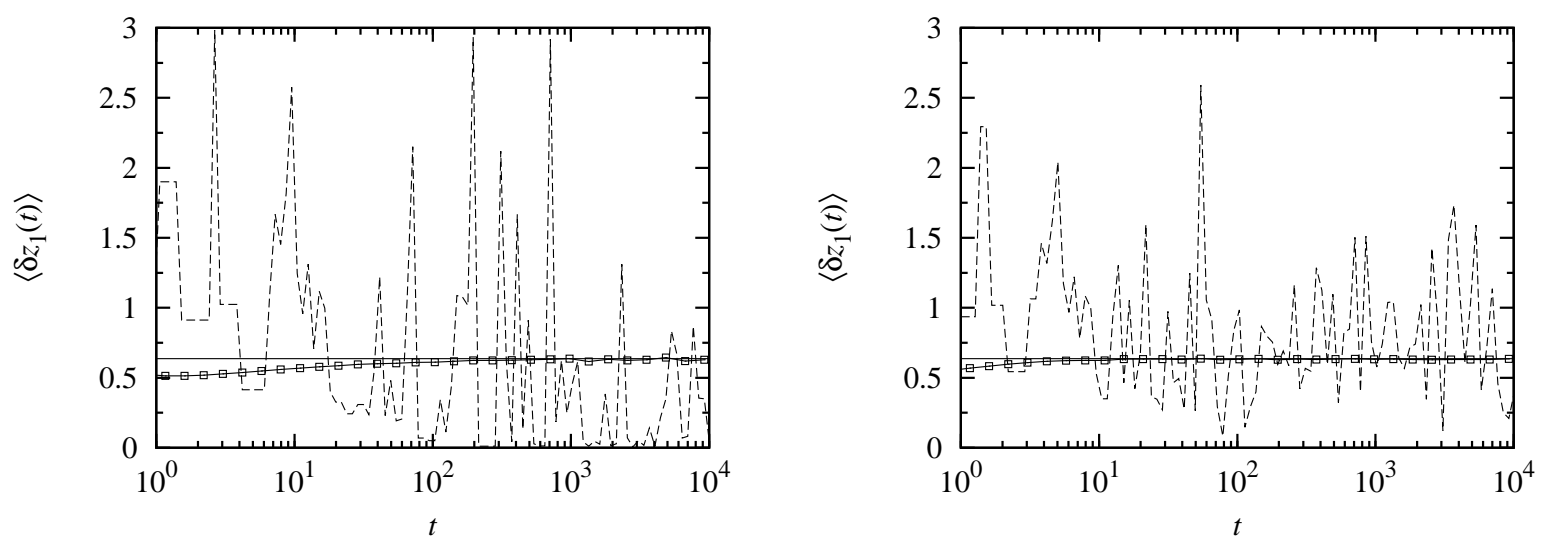

FIG. 2. (Symbols) Average elongation along the streamline for (left panel) $\beta=3 / 2$ and (right panel) $\beta=5 / 2$. (Dashed) Evolution of elongation in a single realization for $z_{1}(0)=1$ and $z_{2}(0)=0$. The horizontal solid lines indicates the asymptotic values (36).

Its Laplace transform is given by

$$
\begin{aligned}
C_{v}(\lambda) & =\sum_{n=0}^{\infty}\left\langle v_{0} v_{n} \exp \left(-\lambda t_{n}\right) \frac{1-\exp \left(-\lambda \tau_{n}\right)}{\lambda}\right\rangle \\
& =\frac{1-\psi(\lambda)}{\lambda}+\left\langle v_{0} \exp \left(-\lambda \tau_{0}\right)\right\rangle \sum_{n=1}^{\infty} \psi(\lambda)^{n-1}\left\langle v_{n} \frac{1-\exp \left(-\lambda \tau_{n}\right)}{\lambda}\right\rangle \\
& =\frac{1-\psi(\lambda)}{\lambda}+s_{0}^{2} \frac{\left\langle\tau_{0}^{-1} \exp \left(-\lambda \tau_{0}\right)\right\rangle}{1-\psi(\lambda)}\left\langle\tau^{-1} \frac{1-\exp (-\lambda \tau)}{\lambda}\right\rangle \\
& =\frac{1-\psi(\lambda)}{\lambda}+\frac{s_{0}^{2}}{\lambda} \frac{\left\langle\tau_{0}^{-1} \exp \left(-\lambda \tau_{0}\right)\right\rangle}{1-\psi(\lambda)}\left[\frac{\langle v\rangle}{s_{0}}-\left\langle\tau^{-1} \exp (-\lambda \tau)\right\rangle\right]
\end{aligned}
$$

In the limit of $\lambda \ll 1$, we obtain

$$
C_{v}(\lambda) \approx \frac{1}{\lambda}\left\langle v_{0}\right\rangle \frac{s_{0}}{\langle\tau\rangle} .
$$

Thus, the asymptotic long time value is given by

$$
\lim _{t \rightarrow \infty} C_{v}(t)=\frac{s_{0}\left\langle v_{0}\right\rangle}{\langle\tau\rangle} .
$$

For the gamma velocity PDF employed in this Letter, we obtain from (26) and (27)

$$
\lim _{t \rightarrow \infty}\langle v(0) v(t)\rangle=v_{c}^{2} \frac{\Gamma(\beta+1)}{\Gamma(\beta-1)} .
$$

\section{NUMERICAL SIMULATIONS}

The numerical Monte-Carlo simulations illustrating the average strip elongation in Figure 2 model the streamwise velocity PDF by the Gamma distribution

$$
p_{v}(v)=\frac{\left(v / v_{c}\right)^{\beta-1} \exp \left(-v / v_{c}\right)}{v_{c} \Gamma(\beta)},
$$

which yields for the transition time distribution

$$
\psi(\tau)=\frac{1}{\tau_{v} \Gamma(\beta)} \frac{\exp \left(-\tau_{v} / \tau\right)}{\left(\tau / \tau_{v}\right)^{1+\beta}} .
$$

We set $v_{c}=1$ and $s_{0}=1$ such that $\tau_{v}=1$. The initial strip orientation angle $\phi$ is uniformly distributed in $[-\pi / 2, \pi / 2]$. 

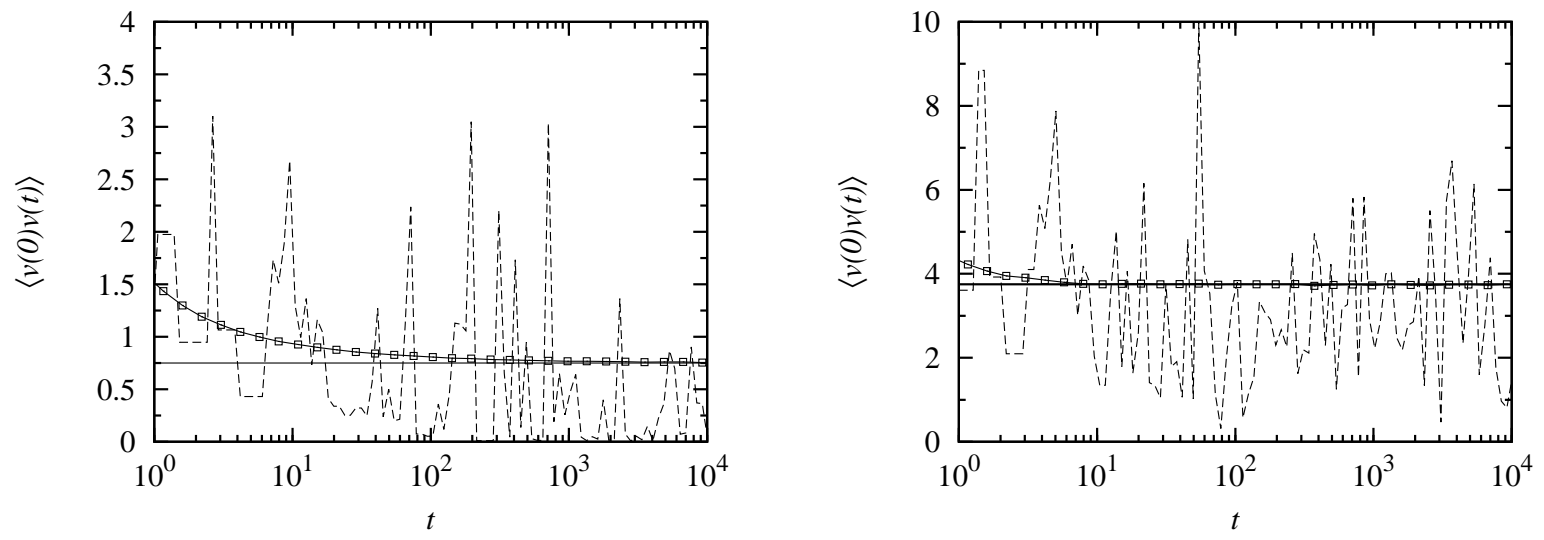

FIG. 3. (Symbols) Average velocity cross-product for (left panel) $\beta=3 / 2$ and (right panel) $\beta=5 / 2$. (Dashed) Evolution of the cross-product in a single realization for $z_{1}(0)=0$ and $z_{2}(0)=1$. The horizontal solid lines indicate the asymptotic values (44). 